\title{
Les cellules souches hématopoïétiques ne résistent pas au chant de la sirène SDF-1
}

Une nouvelle de médecine/sciences évoquait récemment l'importance de la migration des cellules souches et des progéniteurs hématopoïétiques pour leur fonction, et soulignait le contraste entre le peu de connaissances des mécanismes en cause et l'abondance de données sur ceux qui régissent la circulation des leucocytes ( $m / s$ 1999, $n^{\circ} 2$, p. 295). Pourtant, la migration des cellules souches hématopoiétiques est cruciale au cours de l'ontogenèse, assurant les échanges entre les différents sites anatomiques de production des cellules hématopoiétiques. Elle l'est aussi après transplantation de cellules souches hématopoiétiques, puisque la restauration d'une hématopoïèse à long terme dans la moelle du receveur requiert que les cellules du donneur migrent jusqu'à la moelle, traversent la barrière endothéliale, et établissent des interactions stables avec les cellules stromales du receveur dans le microenvironnement médullaire.

Intégrines et sélectines assurent en partie ces processus in vivo, comme le montre l'invalidation du gène de l'intégrine $\beta 1$, mais si on suspectait que les chimiokines étaient des partenaires incontournables, encore fallait-il le démontrer. C'est chose faite pour SDF-1 (stromal cell-derived factor1, anciennement PBSF pour Pre-B-cell growth-stimulating factor, en raison de son activité sur les cellules pré-B) [1], grâce aux travaux du groupe de T. Lapidot (Institut Weizmann, Rehovot, Israël) [2]. SDF-1 exerce une activité chimio-attractante puissante sur les lymphocytes, les monocytes et les cellules primaires $\mathrm{CD}^{+} 4^{+}$[3] par l'intermédiaire de son récepteur CXCR4, plus connu pour son rôle comme co-récepteur du VIH $(\mathrm{m} / \mathrm{s}$
1997, $n^{\circ} 2$, p. 264). L'invalidation de $S D F-1$ est létale en raison d'anomalies cardiaques, mais les anomalies hématologiques sont évidentes: le nombre de précurseurs pro-B dans le foie foetal et la moelle est bas, alors que le développement lymphoïde T est intact [4]. Surtout, la moelle ne contient que de rares cellules myéloïdes, alors que le déficit est beaucoup moins prononcé dans le foie fœetal, ce qui pouvait suggérer une anomalie de migration des progéniteurs hématopoiétiques du foie vers la moelle. Trois groupes ont rapporté des anomalies hématologiques très similaires chez les souris CXCR4 ${ }^{-/-}$ auxquelles s'associent des altérations cardiaques et de la formation du cervelet [5-7]. Cette similarité dans le phénotype des souris $C X C R 4^{-/-}$et $S D F-1^{-/}$démontre que CXCR4, contrairement à la majorité des autres récepteurs aux chimiokines, n'a qu'un seul ligand, SDF-1, et viceversa. Ces résultats, et les observations obtenues in vitro dans des tests de migration, faisaient de SDF-1 la première chimiokine (et actuellement la seule) exerçant, tout au moins in vitro, une action chimioattractante sur les cellules hématopoïétiques primitives. Troisième argument, SDF-1 est synthétisé majoritairement par les cellules stromales médullaires.

Les résultats publiés par le groupe de T. Lapidot plaident en faveur du rôle de SDF-1/CXCR4 in vivo. Si on injecte à des souris NOD-SCID des cellules $\mathrm{CD}^{+} 4^{+}$de sang de cordon préincubées avec un anticorps neutralisant le site de liaison pour SDF-1 de CXCR4, l'implantation de ces cellules dans la moelle des souris est très diminuée. Corroborant ces observa- tions, les auteurs démontrent que les cellules qui migrent in vitro en réponse à SDF-1 à travers la membrane d'une chambre de Boyden ont une efficacité de transplantation supérieure aux cellules qui n’ont pas migré. T. Lapidot montre aussi que les propriétés chimio-attractantes attribuées à certaines cytokines comme le SCF (stem cell factor) sont indirectes et s'expliquent par l'induction du récepteur CXCR4 par ces cytokines. Il existe en effet une corrélation entre l'expression de CXCR4 après traitement par des cytokines et l'efficacité du développement hématopoiétique dans la moelle des souris transplantées, y compris dans des expériences de transplantation secondaire, qui explorent un compartiment de cellules souches très primitives. Même si le critère utilisé par les auteurs (présence de cellules humaines dans la moelle des souris) ne permet pas de distinguer ce qui revient au homing proprement dit et à la prolifération intramédullaire, il est probable que l'expression de CXCR4 soit essentielle pour la prise de greffe des cellules humaines dans les souris NOD/SCID. Ces résultats confirment les données obtenues in vitro, et incitent à considérer SDF-1 comme un maillon important de la cascade des molécules impliquée dans ce processus de migration/colonisation. L'extrapolation à une situation de transplantation humaine est tentante: par exemple l'interruption de l'interaction de SDF-1 avec son récepteur CXCR4 pourrait être impliquée dans le passage des cellules primitives de la moelle vers le sang périphérique après traitement chimiothérapique et/ou traitement par des cytokines (processus de 
mobilisation). Par extension cette interaction pourrait également expliquer le passage des cellules leucémiques dans le sang.

F.L.

1. Nagasawa T, Nakajima T, Tachibana $\mathrm{K}$, et al. Molecular cloning and characterization of a murine pre-B-cell growth-stimulating factor/stromal cell-derived factor 1 receptor, a murine homolog of the human immunodeficiency virus 1 entry coreceptor fusin. Proc Natl Acad Sci USA 1996 ; 93 : 14726-9.

2. Peled A, Petit I, Kollet O, et al. Dependence of human stem cell engraftment and repopulation of NOD/SCID mice on CXCR4. Science 1999; 283: 845-8.

3. Aiuti A, Webb IJ, Bleul C, Springer T, Gutierrez-Ramos JC. The chemokine SDF-1 is a chemoattractant for human CD34 ${ }^{+}$hematopoietic progenitor cells and provides a new mechanism to explain the mobilization of $\mathrm{CD} 34^{+}$progenitors to peripheral blood. J Exp Med 1997; 185 : 111-20. 4. Nagasawa T, Hirota S, Tachibana $\mathrm{K}$, et al. Defects of B-cell lymphopoiesis and bone marrow myelopoiesis in mice lacking the CXC chemokine PBSF/SDF-1. Nature 1996; 382: 635-8.

5. Ma Q, Jones D, Borghesani, P, et al. Impaired Blymphopoiesis, myelopoiesis, and derailed cerebellar neuron migration in CXCR4- and SDF-1-deficient mice. Proc Natl Acad Sci USA 1998; 95: 9448-53.

6. Tachibana K, Hirota S, Lizasa H, et al. The chemokine receptor CXCR4 is essential for vascularization of the gastrointestinal tract. Nature 1998; 393: 591-4.

7. Zou YR, Kottmann A, Kuroda M, Taniuchi I, Littman DR. Function of the chemokine receptor CXCR4 in haematopoiesis and in cerebellar development. Nature 1998; 393: 595-9.

\section{口 BRÈVES}

Survenue de cancers après traitement par la ciclosporine... rôle d'une secrétion accrue de TGF- $\beta$ ? La ciclosporine, utilisée dans le traitement des maladies auto-immunes et la prévention du rejet de greffes, expose, comme toute thérapeutique immunodépressive, à un risque accru de cancers, par défaut de surveillance immune. Dans la cellule, la ciclosporine se lie à la cyclophiline, et le complexe inhibe l'action de la calcineurine, qui active les facteurs NF-AT (nuclear factor of activated $T$ cells) et permet leur translocation nucléaire. Il en résulte le blocage de la transcription des gènes des cytokines effectrices de la réponse immune, dont l'IL-2. Curieusement, ni l'invalidation du gène de l'IL-2, ni celle du gène de NF-AT, ne miment complètement les effets de l'administration de ciclosporine. Hojo et al. [2], démontrent que la ciclosporine a un effet majeur sur la synthèse de TGF- $\beta$ et suggèrent son rôle déterminant dans la survenue de cancers après un traitement prolongé par la ciclosporine. On sait que la ciclosporine stimule la transcription du gène codant pour le TGF- $\beta$ et que cette cytokine favorise l'acquisition d'un phénotype invasif par des cellules $[3,4]$. L'article de Nature montre que le traitement in vitro, par la ciclosporine de cellules déjà transformées leur confère des propriétés caractéristiques de cellules métastatiques, notamment la capacité de proliférer en l'absence de substrat (anchorage independent growth), et l'extension de pseudopodes témoignant d'une motilité accrue qui a été confirmée dans un test de migration in vitro. La survenue, chez des souris SCID/beige traitées par la ciclosporine, d'un nombre deux fois plus élevé de métastases pulmonaires après injection de cellules tumorales confirme que, en dehors de tout contexte d'immunosurveillance, la ciclosporine peut aggraver le caractère invasif de cellules tumorales et ce par le biais d'une sécrétion de TGF- $\beta$. Les données sont assez convaincantes mais, dans ce modèle, les cellules sont déjà transformées. Pourrait-on incriminer la responsabilité de la ciclosporine dans l'accélération de l'émergence d'une tumeur non détectable ou la progression de cellules précancéreuses vers un phénotype tumoral, ou la conversion d'une tumeur bénigne? Reste aussi à comprendre si les autres effets secondaires de la ciclosporine, en particulier sur les tissus hépatique, rénal ou cutané, de mécanisme encore obscur, pourraient aussi être expliqués par une hypersecrétion de TGF- $\beta$.

[1. Baumann G, et al. Med Sci 1992; 8: 366-71.]

[2. Hojo M, et al. Nature 1999; 397 : 530-4.]

[3. Welch DR, et al. Proc Natl Acad Sci USA 1990; 87 : 7678-82.]

[4. Cui W, et al. Cell 1996; 86: 53142.] 